\title{
EINLEITUNG: ZUM SPANNUNGSFELD \\ VON ETHIK UND WIRTSCHAFT
}

Hans G. Nutzinger

\section{Wirtschaftsethik zwischen Akklamation und moralischer Verdammung}

Zumindest in der neueren Geschichte waren die Beziehungen zwischen Ethik und Wirtschaft schon immer konfliktträchtig. In der gegenwärtigen Diskussion scheint sich, veranlaßt durch dramatische aktuelle Entwicklungen, dieser Konflikt noch zuzuspitzen: Bedrohliche weltweite Rüstungsproduktion und Rüstungsgeschäfte, Uberschuldung und wachsendes Elend in der Dritten Welt, die globale Gefährdung unserer natürlichen Lebensgrundlagen - das sind nur einige der Themen, die berechtigten Anlaß zur - leider nicht immer von Sachkenntnis getragenen - Kritik an unserer Art des Wirtschaftens geben. Eine ethisch begründete Neuorientierung der Ökonomie wird gefordert, und sie ist auch in der Tat erforderlich ${ }_{\aleph}$ Nun empfinden Verantwortliche in der Wirtschaft und der Politik oftmals solche ethischen Forderungen als von außen an sie herangetragene "Zumutungen", die den komplexen ökonomischen und politischen Erfordernissen hochgradig arbeitsteiliger und weltweit miteinander verbundener Gesellschaften in keiner Weise gerecht werden können. Zu Recht wird geltend gemacht, daß jede ernstzunehmende Kritik zunächst einmal die funktionalen Zusammenhänge des kritisierten Gegenstandes hier der Wirtschaft - zur Kenntnis zu nehmen habe. Gewiß ist dies nicht immer in ausreichendem Maße der Fall. Allerdings entsteht in der Öffentlichkeit oftmals weit über den engeren Kreis der engagierten Kritiker unserer Wirtschaftsweise hinaus - der Eindruck, daß derartige Verweise auf wirtschaftliche Sachgesetzlichkeiten nicht selten dazu dienen sollen, daß sich die Verantwortlichen in Wirtschaft und Politik ethischen Fragestellungen gänzlich zu entziehen trachten.

Max Webers (1968, S.175) polarisierende Gegenüberstellung einer "religiösen" Gesinnungsethik und einer handlungs- und folgenbezogenen Verantwortungsethik erlebt also unter neuem Vorzeichen eine gefährliche Renaissance. ${ }^{1}$ Max Webers Auffassung, es sei "ein abgrundtiefer Gegensatz, ob man unter der gesinnungsethi-

1 Vgl. dazu im vorliegenden Sammelband den Beitrag von Wilhelm Krelle und die kritische Reaktion von Eberhard Stock darauf. 
scnen Maxime handelt - religiös geredet: 'Der Christ tut recht und stellt den Erfolg Gott anheim' - oder unter der verantwortungsethischen: da B man für die (voraussehbaren) Folgen seines Handelns aufzukommen hat", wird erneut in polemischer Absicht bemüht: Die verantwortlichen Akteure in Wirtschaft und Politik sehen sich selbst of tmals als die sachbezogenen Verantwortungsethiker und ihre Kritiker als die wohlmeinenden, aber inkompetenten Gesinnungsethiker. In dieser holzschnittartigen Selbstwahrnehmung vieler "Macher" steckt zweifellos ein Körnchen Wahrheit. Beschädigt wird allerdings dieses bequeme Selbstbild dadurch, daß es oftmals gerade diese "Verantwortlichen" in den Führungsrängen von Wirtschaft und Politik sind, die, einmal auf ihre individuelle Verantwortung für die von ihnen (mit-) veranlaßten Handlungskonsequenzen angesprochen, sich allzuschnell und allzuleicht als Gefangene eines sozialen Organismus darstellen, der ihnen ihr Handeln gleichsam diktiert und praktisch kaum mehr Raum für individuelle Verantwortung läBt. So entpuppen sich manche selbsternannten Verantwortungsethiker im Ernstfall als Akteure eines komplexen wirtschaftlichen und politischen Beziehungsgeflechts, die - auf ihr persönliches Eintreten für die von ihnen verursachten Folgen hin befragt - gewissermaßen argumentative "Nebelkerzen" werfen in der Hoffnung, angesichts der komplizierten Interdependenz menschlicher Handlungen, die keine einfachen kausalen Ursache-Wirkungs-Relationen zuläßt, löse sich ihre individuelle Verantwortung in einem (teils selbsterzeugten) diffusen sozialen $\mathrm{Ne}$ bel auf. Von der Pose der Verantwortung über die Behauptung der sozialen Determiniertheit individuellen Verhaltens ist es dann oft nur noch ein kurzer Weg bis hin zur Zurückweisung persönlicher Verantwortung.

Derartige Entlastungsversuche mancher Betroffenen scheitern oftmals schon daran, daB man ihnen durchaus Handlungsspielräume - und damit auch Möglichkeiten der Wahrnehmung oder Verfehlung individueller Verantwortlichkeit nachweisen kann. Aber selbst wenn und soweit derartige Handlungsspielräume nicht bestehen, ist damit die ethische Anfrage an das Wirtschaften noch nicht hinauseliminiert: Sie verlagert sich dann zu einer Frage nach der ethischen Qualität bestehender Wirtschafts- und Gesellschaftsordnungen. Es sind dann nicht mehr die individuellen Akteure, die man gegebenenfalls an ihren Früchten als "falsche Propheten" erkennen kann (vgl. Matthäus 7, 16), sondern die wirtschaftlichen und politischen Ordnungen, in denen diese Akteure leben, die man nach ihren erkennbaren Konsequenzen für alle Beteiligten ethisch zu beurteilen hat. Angesichts weltweit bedrückender Entwicklungen - wir hatten beispielhaft Problembereiche wie 
Rüstung, Dritte Welt und Umweltgefährdung genannt - ist die Notwendigkeit, aber auch die Verlockung einer ethischen (Ab-)Qualifizierung dieser Ordnungen besonders groß.2 Die Notwendigkeit einer ethischen Beurteilung sozialer Ordnungen ist gerade angesichts eines komplizierten wechselseitigen Zusammenhangs wirtschaftlicher Entscheidungen, Handlungen und Konsequenzen, der oftmals keine eindeutigen Adressaten individueller Verantwortlichkeit zuläßt, unmittelbar einsichtig. Ebenso verständlich ist auch die Versuchung gerade für den Ethiker oder den Theologen, sich erst gar nicht auf die Analyse dieser komplexen Interdependenzen einzulassen und statt dessen, gestützt auf offenkundig unannehmbare Konsequenzen dieser ökonomischen Prozesse, die gesamte Wirtschafts- und Gesellschaftsordnung moralisch abzuqualifizieren. Ein derartiges Vorgehen hat aber zumindest drei Nachteile:

- Der ethisch argumentierende Kritiker wird von den unmittelbaren Akteuren in der Wirtschaft kaum als Dialogpartner anerkannt, da er ja offenkundig "nichts von Wirtschaft versteht". Er erscheint dann oftmals als der externe Kritikaster, der in handlungsentlasteten Situationen und Institutionen - wie Hochschule oder Kirche - lebt und "gut reden hat".

- Das Fehlen einer sachkundigen Analyse führt überdies häufig dazu, daß eine falsche Zurechnung unerwünschter Ergebnisse auf die sie angeblich verursachenden Ordnungselemente erfolgt; so werden 2.B. weltweite Umweltschäden oder Überschuldung in Ländern der Dritten Welt mitunter pauschal "der Marktwirtschaft" zugeschrieben, obwohl eine eingehendere Analyse zeigt, daB es oftmals gerade das Fehlen marktwirtschaftlicher Steuerungsmechanismen war, das zu dem beklagten Zustand beigetragen hat. ${ }^{3}$

- Den Kritikern bestehender Wirtschafts- und Gesellschaftsordnungen gelingt es aufgrund ihres häufig mangelhaften Fachwissens regelmäßig nicht, die von ihnen geforderte "andere Wirtschaftsordnung" hinreichend zuverlässig und präzise zu beschreiben.

2 Vgl. dazu auch die in dem Beitrag von Hermann Sautter eingangs angefūhrten Beispiele.

3 Vgl. dazu die Ausführungen in den Beiträgen von Sautter und Nutzinger. 
Man sieht also, eine ernstzunehmende Wirtschaftsethik muß eine schwierige Gratwanderung zwischen zwei gleichermaßen bedrohlichen Abgründen meistern: Auf der einen Seite muß sie vermeiden, sich nur bestätigend auf vermeintliche oder tatsächliche Funktionsnotwendigkeiten einzulassen und damit sozusagen die moralische Sauce einzurühren, in der dem Unternehmer der Gewinn, dem Arbeitnehmer seine Weihnachtsgratifikation und dem Hungernden in der Dritten Welt seine Lebensmittelspende eingerührt wird. Zum anderen muß sie sich bei ihren ethischen Bewertungen wirtschaftlicher und politischer Zusammenhänge immer wieder an den funktionalen Erfordernissen dieser Bereiche orientieren, ohne daß sie sich damit auch das Recht zu einer umfassenden ethischen Bewertung bestehender Wirtschafts- und Gesellschaftsordnungen nehmen läßt. Der Wirtschaftsethiker muB also, wie der Pilger auf dem schmalen Grat zum Heil, die Gefahr der ethischen Akklamation bestehender Ordnungen ebenso vermeiden wie die ihrer moralisierenden Pauschalverdammung.

Die vorangegangenen Überlegungen lassen sich in zwei zentralen Leitfragen zusammenfassen:

1. Inwieweit gibt es einen Bereich "ökonomischer Sachgesetzlichkeiten", den die Ethik zu respektieren hätte, und inwieweit muß auch andererseits die "Wirtschaft" ethische Forderungen gegen sich gelten lassen, die sie nicht einfach unter Hinweis auf wirtschaftliche Funktionszusammenhänge von vornherein zurückweisen kann?

2. Wie können im Zusammenwirken aller Beteiligten reale Bedingungen geschaffen werden, die verantwortliches ethisches Handeln in der wirtschaftlichen Realität unserer Zeit ermöglichen, so daß Ethik weder ein bloßer moralischer Appell an individuelles Verhalten noch eine - positive oder negative - Pauschalbewertung wirtschaftlicher und gesellschaftlicher Ordnungen "von außen" bleibt?

Die im folgenden wiedergegebenen Beiträge eines Expertengesprächs von Theologen, Philosophen, Ökonomen und Wirtschaftspraktikern widmen sich diesen beiden Leitfragen und untersuchen diese auch anwendungsbezogen anhand von drei Beispielen, nämlich der Frage von Ethik im Unternehmen, am Problem der Ver- 
schuldung vieler Entwicklungsländer und schließlich am Beispiel der globalen Umweltproblematik.

\section{Die Beitrāge im einzelnen}

\subsection{Positionsbestimmungen der Wirtschaftsethik}

Der Band beginnt mit zwei grundsätzlichen Beiträgen, die das Verhältnis von Ethik und Wirtschaft in philosophischer und in theologischer Sicht beleuchten. Der Hamburger Philosoph Josef Meran untersucht zunächst die Rolle der Wirtschaftsphilosophie als Wirtschaftsethik und als Wissenschaftstheorie der ökonomischen Wissenschaft. Sodann setzt er sich mit zwei - bewußt stark stilisierten - Auffassungen über das Verhältnis von Moral und Wirtschaft auseinander, nämlich zum einen mit der Vorstellung, da $B$ zwischen wirtschaftlichem und moralischem Handeln keine Beziehung bestehe, gestützt durch eine Form der Wirtschaftsphilosophie, die ihre Aufgabe darin sieht, die Freisetzung wirtschaftlichen Handelns von moralischer und religiöser Bevormundung ethisch zu rechtfertigen. In diesen Entwürfen für sie stehen so berühmte Namen wie John Locke, Adam Smith und in neuerer Zeit Friedrich August von Hayek - wird, wenn auch mit unterschiedlicher Stärke, die Rolle der wirtschaftlichen Eigengesetzlichkeiten betont. Diesem mehr oder minder pointiert vertretenen "Rechtfertigungsmodell" der Wirtschaftsethik steht seit spätestens Aristoteles eine Tradition der "moralischen Ökonomie" gegenüber, die wirtschaftliches Handeln mehr oder minder vollständig moralischen Normen und Handlungsanweisungen unterordnen will.4 ${ }^{4}$ dieser moralischen Tradition wird eine besondere Sachgesetzlichkeit insbesondere dann in Frage gestellt, wenn die ihr entspringenden Ergebnisse dem eigentlichen ethischen Ziel des Wirtschaftens - nämlich dem sittlich "guten Leben" des Menschen in der Gemeinschaft - widersprechen. Da sich in diesen Gegenentwürfen das wirtschaftliche Handeln und die Wirtschaftsordnung moralischen Normen zu beugen hat, spricht Josef Meran in bewußter Überspitzung auch von einem "Unterdrückungs-Modell" der Wirtschaftsethik.

Sodann bestimmt Meran die zunehmende Ökonomisierung unserer öffentlichen und privaten Lebensbereiche als neuen Ausgangspunkt einer motivationalen Wirt-

Vgl. zu dieser "moralischen Tradition" insbesondere den Beitrag von Eberhard K. Seifert im vorliegenden Sammelband. 
schaftsethik, die ihre Aufgabe primär nicht darin sieht, ein universales - auch für das Wirtschaften geltende - Moralprinzip zu begründen, sondern darzulegen, wie ökonomische und moralische Motive und Normen in Einklang mit wirtschaftlichen Funktionszusammenhängen gebracht werden können. Demzufolge soll Wirtschaftsethik nicht "von außen" die vorhandene (markt-)wirtschaftliche Ordnung moralisch (ab-)qualifizieren, sondern gerade an das Tauschprinzip des marktwirtschaftlichen Handelns anknüpfen und dem "homo oeconomicus" klarmachen, daß es durchaus ökonomisch vernünftig ist, auch moralisch richtig zu handeln. Meran plädiert also für eine Klugheitsethik, die der Vermittlung der Einsicht dient, daß ein moralisches Handeln der Menschen im Interesse ihrer wahren, d.h. im Einklang mit ihren eigenen Glücksvorstellungen befindlichen, Bedürfnisse liegt.

Der Erlanger Theologe Hans G. Ulrich beleuchtet anschließend theologische $\mathrm{Zu}$ gänge zur Wirtschaftsethik. Als einen besonders wichtigen Ansatzpunkt theologischer Reflexion ökonomischer Probleme kennzeichnet er die Frage nach einem "realistischen" Bild vom Menschen, in dem es auch Platz für das strukturell "Böse" gibt, das nicht berechenbar und durch Vertragsklauseln beherrschbar ist. Leitfrage der Wirtschaftsethik ist für Ulrich nicht, ob und wie sich Moralität im Wirtschaftsleben "auszahlt", sondern die Frage, wie die Wirtschaft und die Praxis des Zusammenlebens (die Lebenswelt) zusammengehören und koexistieren. Als zentrales Moment des christlichen Ethos sieht er das "Ethos der Hoffnung", getragen von dem Vertrauen auf die Verheißung Gottes. Demzufolge wendet er sich gegen eine verkürzte Ethik, die sich etwa auf die Diskussion von Handlungsfolgen oder -zielen und deren Begründung beschränkt, und plädiert statt dessen für eine weiter gefaßte Ethik, die auch die sozialen Vorgänge und Handlungszusammenhänge reflektiert, innerhalb derer Ökonomie stattfindet; damit kommt Ulrich auch zur klassischen Frage nach dem Zusammenhang zwischen dem wirtschaftlichen "Nutzen" und dem für das Zusammenleben notwendigen "Guten". Er spricht in diesem Zusammenhang von der "Ökonomie Gottes", in der dem Menschen als Teil der Schöpfung Güter gegeben sind, die er empfangen und annehmen darf. Der Mensch braucht demzufolge nicht nur verschiedene (Wirtschafts-)Güter, "sondern er ist selbst der Rettung bedürftig" (S. 48 unten).

So wichtig für Ulrich die ökonomische Rationalität als Maßstab ökonomischen Erfolges ist, so sieht er auch noch Rationalitäten anderer Lebensbereiche, die mit der ökonomischen Rationalität zu koordinieren - und nicht einfach gleichzusetzen - 
sind. Als Hauptgegenstände wirtschaftsethischer Reflexion benennt er die Bereiche "Arbeit", "Eigentum" und "Umwelt", die ja auch zugleich zentrale Kategorien der ökonomischen Theorie sind. Daher kommt der Wirtschaftsethik auch eine theoretische Aufgabe zu, nämlich die Auseinandersetzung mit der Frage, "wie der Mensch, der mit Gott lebt, mit der Ökonomie koexistieren kann, und wie die ökonomische Theorie mit ihm zu 'rechnen" vermag (unten S.56f.). Da eine theologisch reflektierte Wirtschaftsethik auf die Praxis des Wirtschaftens als einer Praxis von Menschen zielt, die zugleich in anderen Bereichen menschlicher Praxis leben, geht es nicht darum, eine Universaltheorie praktischen Handelns zu entwerfen, sondern darum, Koexistenzformen dieser verschiedenen Bereiche, auch auf unterschiedlichen Ebenen, zu gewinnen, und zwar "unter Einschluß der Widersprüche, die nicht aufzulösen sind" (unten S.57). Deswegen soll der Mensch von der Ethik nicht einfach vereinnahmt, sondern in seiner Geschöpflichkeit, in seinem Verhältnis zu Gott ernst genommen werden.

Sodann beleuchtet Eberhard K. Seifert (Hamburger Institut für Sozialforschung) das Verhältnis von Ökonomie und Ethik in historischer und dogmenhistorischer Perspektive. Ihm geht es vor allem um die Wiederbelebung jener seit der Antike bestehenden Denktradition, die Josef Meran zuvor als "moralische Ökonomie" bezeichnet hatte. Anders als bei ihm ist sie für Seifert aber kein "UnterdrückungsModell" der Wirtschaftsethik, sondern eine wichtige, zu Unrecht in den Hintergrund gedrängte Richtung ökonomischen Denkens. Die aristotelische Tradition, die er für die Gegenwart neu beleben möchte, gilt ihm als Beleg dafür, daß die Beziehungen zwischen Ethik und Wirtschaft nicht immer schon konfliktträchtig waren. Auch für Adam Smith, den Begründer der modernen Wirtschaftswissenschaft, sieht Seifert noch eine - wenn auch nicht mehr aristotelisch begründete - Einheit von Ethik und Ökonomik, die er in der "historisch-ethischen" Schule Gustav von Schmollers erneut thematisiert sieht. Seifert betont den Beitrag dieser Denkrichtung zur Lösung der damals drängenden "socialen Frage" und sieht als Hauptgrund dafür, daß die angelsächsische, neoklassisch orientierte Ökonomik auch in Deutschland vorherrschend wurde, den Umstand, daß die von Schmoller unterstellte sozialreformerische "Ethik des Staates" durch die späteren Erfahrungen mit autoritären und faschistischen Perversionen staatlicher Gewalt, zumal in Deutschland, unwiderruflich diskreditiert seien. So sympathisch ein derartiger Erklärungsansatz sein mag, er greift doch angesichts der analytischen Defizite der historischethischen Schule meines Erachtens eindeutig zu kurz. 
An diese generellen Darlegungen zum Verhältnis von Ökonomie und Ethik schließt sich nun eine Auseinandersetzung um die Unterscheidung zwischen "positiver und negativer Ethik" an, die der Bonner Nationalökonom Wilhelm Krelle zum Ausgangspunkt seines Beitrags macht: Mit dem Alten Testament verbindet er primär eine handlungseinschränkende Verbotsethik, wie wir sie insbesondere aus dem Dekalog kennen, während er im Neuen Testament vor allem handlungseröffnende Gebote, "Du-sollst"-Sätze, sieht. Die letzteren sind Wilhelm Krelle zufolge der Ökonomie näherliegend, weil sie Abwägungen zwischen verschiedenen Alternativen erlauben und insbesondere an die Stelle eines "EntwederOder" ein quantitatives "Mehr oder Weniger" setzen. Diese Überlegung wird schlieblich in einem einfachen formalen Modell am Beispiel der Umweltproblematik (umweltschädliche Güter und Faktoren) verdeutlicht: Ein ethisches Verbot (z.B. der Umweltverschmutzung) wirkt im allgemeinen nutzenmindernd, weil jetzt nicht mehr alle technischen Produktionsmöglichkeiten realisiert werden können; dies braucht im Falle von Geboten (hier: der Umweltschonung) nicht zu gelten, da sich mun die Präferenzen so verändern können, "daß überhaupt kein Nutzenverlust eintritt und trotzdem die Verschmutzungsgrenzen eingehalten werden" (unten S.104). Diese Analyse ist sicherlich zutreffend, allerdings ist die zugrunde gelegte Annahme - Verbote könnten eine derartige Umorientierung der menschlichen Wertvorstellungen nicht erreichen - offenkundig ihrerseits der Kritik zugänglich und bedürftig. Indessen wird man vom ökonomischen Standpunkt aus dem generellen Plädoyer Wilhelm Krelles für mehr Möglichkeiten der Abwägung - oder wirtschaftspolitisch gewendet: für einen verstärkten Einsatz ökonomischer Instrumente des Umweltschutzes - beipflichten müssen, wenn und soweit dadurch natürliche Lebensgrundlagen nicht zerstört oder nachhaltig gefährdet werden.

In der Diskussion dieses Beitrags wurden sowohl von ökonomischer wie von theologischer Seite erhebliche Zweifel an der Leistungsfähigkeit dieser Unterscheidung vorgetragen, die im vorliegenden Band aus theologischer Sicht von Eberhard Stock (Universität Marburg) formuliert sind: Er macht vor allem geltend, da B zunächst einmal das Begründungsproblem von - positiv oder negativ formulierten - Imperativen ethisch zu lösen sei, bevor man sich der Frage nach deren sprachlicher Formulierung zuwenden könne. Die Distinktion zwischen positiven und negativen Geboten und die daran anschließende Typisierung nach Gebots- und Verbotsèthiken hält Stock für problematisch, unter anderem deswegen, weil Gebote und Verbote sich gleichermaßen im Alten und im Neuen Testament finden und weil auch 
die Verbote des Alten Testaments nicht als absolute Handlungseinschränkungen zu sehen, sondern durchaus auch situativ zu fassen sind. Umgekehrt können auch positiv formulierte Gebote unbedingte, keinerlei Abwägungen zulassende Imperative enthalten. ${ }^{5}$ Stock schließt darüber hinaus einige grundsätzliche Überlegungen zur Charakterisierung evangelischer Ethik an, die insbesondere auch ein gängiges Verständnis der Martin Luther oft vordergründig unterstellten "Zwei-ReicheLehre" relativieren. Die Kontroverse schließt mit einer kurzen Erwiderung von Wilhelm Krelle, in der er unter Verweis auf seine formale Analyse noch einmal die Bedeutung der Unterscheidung von positiven und negativen Imperativen verteidigt. Während Stock der Ansicht war, die Unterscheidung zwischen Gesinnungs- und Verantwortungsethik tauge kaum für eine polemische Überspitzung, betont Krelle in seiner Erwiderung nochmals, daß es vielen gutgemeinten Empfehlungen von "Gesinnungsethikern" gerade an ökonomischem Sachverstand mangele. Allerdings zeigt sich hierin auch eine weitgehende Übereinstimmung zwischen Krelle und Stock: Beide plädieren - wenn auch mit unterschiedlicher Begründung und Begrifflichkeit - dafür, Wirtschaftsethik nicht als eine heteronome Zumutung an ökonomische Theorie und ökonomische Praxis zu betreiben.

\subsection{Wirtschaftsethik in der Praxis}

Der zweite Teil des Sammelbandes beschäftigt sich nunmehr mit der Frage der praktischen Umsetzung ethischer Postulate in verschiedenen Bereichen der Ökonomie. Der jüngst verstorbene Ordinarius für Volkswirtschaftslehre an der Universität Hohenheim, Josua Werner, untersucht zunächst das Spannungsfeld zwischen ethischen Postulaten und wirtschaftspolitischen Gestaltungsmöglichkeiten. Er plädiert - hierin Arthur Rich folgend - für eine umfassende Wirtschaftsethik, deren Normen- und Beurteilungssysteme zwar die Funktionszusammenhänge der Wirtschaft zur Kenntnis nehmen und respektieren, aber gleichwohl eigenständig begründet sind. Nach einer Benennung zentraler sozialethischer Prinzipien - Personalität, Solidarität, Subsidiarität und Partizipation - kennzeichnet Werner die Fragestellung der Wirtschaftsethik im besonderen durch die Aufgabe, sie habe

5 Stock verweist hier z.B. auf die Aufforderung Gottes an das Volk Israel: "... Du sollst den Herm, Deinen Gott, liebhaben von ganzem Herzen, von ganzer Seele und mit aller Deiner Kraft" (2. Mose 6,5). 
eigenständige, auf das menschliche Verhalten im Bereich der Wirtschaft ausgerichtete Normen zu entwickeln. Derartige Normen können sich auf die Arbeitsbedingungen bei der Produktion von Gütern beziehen, auf die Frage der Umweltverträglichkeit von Produktionsprozessen und von Gütern oder auch auf die Frage nach den Instrumenten, die beim Absatz der Güter an die Konsumenten zulässig sind.

Nach der Aufzählung dieser primär unternehmensethischen Fragestellungen widmet sich Werner sodann einer ausführlichen ethischen Bewertung des Sozialproduktbegriffs, vor allem orientiert an den Fragen nach Umfang, Wachstum, Struktur und Verteilung der in dieser zentralen statistischen Maßgröße zusammengefaßten Güter und Dienstleistungen. Bei seiner abschließenden Bewertung der Marktwirtschaft als Institution und des ihr zugrundeliegenden Wettbewerbsprinzips sieht er zwar einerseits Mängel und Kritikpunkte, die nicht nur thematisiert, sondern auch in der praktischen Wirtschaftspolitik berücksichtigt werden müßten, meint aber andererseits, daß aus sozialethischer Sicht der Marktwirtschaft die grundsätzliche Anerkennung nicht versagt bleiben dürfe.

Nach dieser grundsätzlichen Bestimmung des Verhältnisses von Ethik und wirtschaftspolitischen Gestaltungsmöglichkeiten wenden sich die drei folgenden Beiträge dem wohl wichtigsten Bereich unserer Wirtschaftsordnung, nämlich den Unternehmen, zu und fragen in unterschiedlicher Weise, wie hier ethische Prinzipien konkret zu verwirklichen sind. Georges Enderle (Hochschule St. Gallen) beginnt mit grundsätzlichen "Annäherungen an eine Unternehmensethik". Er thematisiert zunächst unterschiedliche Erwartungen und Konzeptionen von Unternehmensethik in der heutigen Diskussion, bevor einige grundlegende Elemente von Unternehmensethik systematisch herausgearbeitet werden. Dies führt ihn zur Frage nach dem moralischen Status des Unternehmens; er plädiert dabei dafür, das Unternehmen als einen moralischer Verantwortung fähigen Akteur zu verstehen, obwohl er durchaus auch die Probleme dieses Ansatzes sieht.

Daran anschließend argumentiert Enderle zugunsten einer klaren Trennung von Unternehmensethik und einer Unternehmer- bzw. Führungsethik. Er betont, daß die Unternehmensethik eine eigenständige Bedeutung auch dann behält, wènn die gesamtwirtschaftlichen Rahmenbedingungen ethisch gerechtfertigt sind. Eine systematische Struktur erlaubt es ihm, Voraussetzungen einer Unternehmensethik zu 
formulieren, die einerseits eine instrumentelle Verkürzung auf rationelle Betriebsabläufe vermeidet, andererseits aber auch die Funktionsbedingungen von Unternehmen in einer Marktwirtschaft anerkennt. Damit werden wichtige Grundlagen für die Unternehmensethik gelegt, die gerade angesichts ihrer zunehmenden Relevanz im ausgehenden 20. Jahrhundert von besonderer Bedeutung sind.

Die beiden folgenden Beiträge stammen von verantwortlichen "Wirtschaftspraktikern", die sich insbesondere der Frage nach einer Umsetzung ethischer Prinzipien im wirtschaftlichen Alltag befassen. Otto Walterspiel (Kali + Salz AG Kassel) beginnt seine Diskussion wirtschaftsethischer Fragen aus unternehmerischer Sicht mit eigenen negativen und positiven Beobachtungen aus der wirtschaftlichen Praxis, um zu zeigen, daß Fragen der Ethik in der Wirtschaft eine wichtige Rolle spielen, vor allem auch aufgrund der gesteigerten Eingriffsmöglichkeiten der Menschen in die natürlichen Lebensgrundlagen.

Grundlage ethischen Handelns ist für Otto Walterspiel die objektive sittliche Ordnung, das natürliche "Sittengesetz", aus dem sich sittliche Normen für einzelne Bereiche menschlichen Händelns und für den einzelnen handelnden Menschen ergeben. Eine praktische Konsequenz dieser Orientierung ist zum Beispiel, daß man auch über die Grenzen wachsender Güterversorgung im Hinblick auf die natürlichen Ressourcen nachdenken und gegebenenfalls entsprechende Begrenzungen vornehmen muß. Eine andere Zentralfrage der Ethik bezieht sich auf die Verteilung der Güter unter den heute lebenden Menschen, aber auch zwischen heutigen und künftigen Generationen. Auf der Ebene des Unternehmens stellen sich für die Verantwortlichen schwierige Fragen nach ethisch vertretbaren Lösungen angesichts oftmals konkurrierender Ansprüche von Kunden, Mitarbeitern, Eigentümern und der Allgemeinheit. Deshalb plädiert Walterspiel für die generelle Norm "Wirtschafte gewissenhaft!" - und zwar in allen Lebensbereichen. Letzter Grund ethischer Normen wie auch ethischen Handelns ist für ihn Gott als Schöpfer dieser Welt, der den Menschen nicht nur eine hohe ethische Verpflichtung auferlegt, sondern ihnen auch seine Barmherzigkeit zusichert.

Der zweite Beitrag von Hellmut Steffens (Siemens AG München/Augsburg) fragt zunächst nach den Partnern eines unternehmensethischen Dialogs und ihren jeweiligen Urteilen und Vorurteilen und sodann danach, was Folgenabschätzung für 
jeden einzelnen heißt. Danach ist ethisches Handeln ein Prozeß des Erkennens und Abwägens verschiedener Alternativen, auch auf der Grundlage eines offenen Dialogs mit anderen Disziplinen und Grundhaltungen; dabei kann jeder ethische Forderungen immer nur in dem Maße wirksam erheben, wie er sie vergleichbar auch gegen sich selbst gelten läßt.

Angesichts unterschiedlicher Wertvorstellungen in einer säkularen Gesellschaft kann es nach Steffens' Einschätzung keine allgemeinverbindliche Unternehmensethik geben, sehr wohl jedoch die vom einzelnen Verantwortlichen zu vertretende Unternehmerethik. Dieser kommt auch eine wichtige Vorbildfunktion für die Mitarbeiter im Unternehmen zu. So können auch durch Mitarbeiterinitiativen gemeinsam ethische Leitlinien entwickelt, vereinbart und beachtet werden. Zentrale Kraftquelle ethischen Handelns ist für Hellmut Steffens aber - jenseits aller wirtschaftlichen Erfolge - das am Glauben des einzelnen orientierte Gewissen.

Ein weiteres Anwendungsfeld der Wirtschaftsethik ist die vom Frankfurter Wirtschaftswissenschaftler Hermann Sautter behandelte Frage der internationalen Schuldenkrise als Beispiel für die wirtschaftsethische Problematik der internationalen Nord-Süd-Beziehungen. Sein Beitrag besticht vor allem durch eine ausgezeichnete inhaltliche Analyse der wirtschaftlichen, sozialen und politischen Situation in den von der Schuldenkrise am meisten betroffenen Entwicklungsländern. Ausgangspunkt seiner wirtschaftsethischen Beurteilung des Schuldenproblems ist zunächst einmal die Feststellung, da $B$ ihre wirtschaftsethische Betrachtung vorwiegend sozialethischer Art sein muß und sich nicht primär auf individualethische Erwägungen stützen kann. Dabei geht Sautter davon aus, daß Interaktionen auf Kreditmärkten nicht per se schon unmoralisch sind, daß Ordnungsregeln für den privatwirtschaftlichen Verkehr in einem gesamtgesellschaftlichen AbstimmungsprozeB bewußt gesetzt werden müssen und $\mathrm{da} \beta$ es durchaus auch individualethische Dimensionen des Problems aufgrund der Entscheidungsspielräume der beteiligten Akteure gibt. Aufgrund dieser Überlegungen kommt Sautter nun einerseits zu einem grundsätzlich positiven Urteil über marktwirtschaftliche Koordinationsmechanismen, aber auch zu dem Ergebnis, daB im untersuchten Fall die erforderlichen Ordnungsregeln - sowohl innerhalb der Gläubiger- wie innerhalb der Schuldnerländer sowie in den internationalen Wirtschaftsbeziehungen insgesamt - nur höchst unvollkommen erfüllt waren. 
Damit hat Sautter ein Kriteriensystem gefunden, das es ihm erlaubt, die Entstehung der Schuldenkrise aus ordnungspolitischer und wirtschaftsethischer Sicht detailliert zu untersuchen und zu beurteilen. Daraus entwickelt er schließlich Ansätze zur Lösung des Schuldenproblems, die zum einen auf einen konditionierten Schuldenerlaß, verbunden mit bestimmten ordnungspolitischen Auflagen, zum anderen auf die Forderung nach ordnungspolitischen Änderungen auch in den Gläubigerländern (z.B. erleichterter Marktzugang für die Entwicklungsländer in den Industrieländern) und auf internationaler Ebene (wirtschaftspolitische Abstimmung und freiwillige Vereinbarungen zwischen den Industrie- und den Entwicklungsländern) hinauslaufen. Auch wenn Sautters Beitrag in vielen Bereichen notwendig allgemein bleibt, liefert er doch ein instruktives Beispiel dafür, daß sich wirtschaftswissenschaftliche Analyse und wirtschaftsethische Beurteilung keineswegs wechselseitig ausschließen müssen, sondern vielmehr höchst sinnvoll miteinander verbinden lassen.

Hans G. Nutzinger behandelt schließlich das Verhältnis von Ökonomie und Ethik am Beispiel der Umweltproblematik. Nach einigen grundsätzlichen Überlegungen zum Spannungsverhältnis von Wirtschaftswissenschaft und wirtschaftlicher Praxis werden unterschiedliche Begründungen zugunsten des Naturschutzes präsentiert und im Anschluß an Hampicke und Birnbacher dafür argumentiert, sich soweit als auf anthropozentrische Begründungen zu beschränken, da diese - wenn man sie weit genug auslegt - durchaus für die praktischen Zwecke des Naturschutzes ausreichen. Im zweiten Teil seiner Überlegungen geht Nutzinger auf die gestiegene Verantwortung an wirtschaftliches und technisches Handeln ein, die sich aus der gesteigerten Reichweite menschlicher Eingriffsmöglichkeiten einerseits und aus dem Vorhandensein eines "strukturell Bösen" in der menschlichen Existenz andererseits ergibt. Zusammenfassend betont er die Notwendigkeit von "ethischer Bildung", zugleich aber auch von Institutionen und allgemeinen Ordnungsregeln, die den Individuen ethisches Handeln ermöglichen und sie nicht individuell überfordern.

Der letzte Beitrag dieses Bandes besteht in der Wiedergabe der Predigt zur Tagung von Akademiedirektor Klaus Röhring über das Thema "Vom unmöglichen und vom möglichen Leben". Die bekannte Geschichte des "reichen Jünglings" erscheint uns hier in neuer Beleuchtung: Deutlich wird, daß es nicht um eine Verdammung von Reichtum schlechthin oder gar um eine Verherrlichung von Armut geht, son- 
dern darum, daß die Erfüllung des Lebens nicht in der Anhäufung von immer mehr Gütern und Vermögen bestehen kann. So endet diese Geschichte in der Ausweglosigkeit, in der Aporie; zugleich aber bleibt auch die Hoffnung des Christen auf Gott, "bei dem alle Dinge möglich sind".

Der Leser, der fertige Rezepte und praktische Handlungsanleitungen in diesem Sammelband sucht, wird vermutlich eher enttäuscht sein. Diese Enttäuschung ist aber weder zufällig noch beabsichtigt, sondern unvermeidlich. Eines machen, so denke ich, die folgenden Beiträge aber deutlich: Wirtschaftsethik braucht nicht zu moralisierender Pauschalkritik oder zur ethischen Überhöhung der bestehenden Wirtschaftsordnung zu verkommen. Sie nimmt aber die Menschen - Christen wie Nicht-Christen - in die Pflicht zu verantwortlichem Handeln. Dazu gehört auf der personalen Ebene das Bedenken der Folgen individuellen Handeins und die Bereitschaft zum Dialog ebenso wie generellere Überlegungen auf der Makroebene darüber, wie bestehende wirtschaftliche und gesellschaftliche Ordnungen so weiterentwickelt werden können, daB nicht nur die allgemeinen Funktionsprinzipien dieser Ordnungen, sondern auch ihre konkreten Ergebnisse ethisch annehmbar sind.

\section{Literatur:}

Weber, Max: "Der Beruf zur Politik", in: ders.: Soziologie - weltgeschichtliche Analysen - Politik. Stuttgart: Kröner, 1968, S. 167-185 